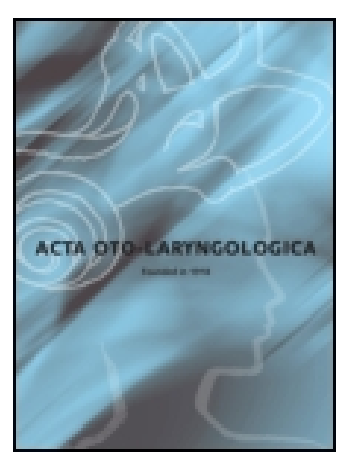

Acta Oto-Laryngologica

\title{
Über Störungen im Harnsäure-Stoffwechsel bei Patienten mit Asthma bronchiale und Rhinitis vasomotoria
}

\section{A. de Kleyn \& W. Storm van Leeuwen}

To cite this article: A. de Kleyn \& W. Storm van Leeuwen (1918) Über Störungen im HarnsäureStoffwechsel bei Patienten mit Asthma bronchiale und Rhinitis vasomotoria, Acta OtoLaryngologica, 1:1, 571-584, DOI: 10.3109/00016481809122018

To link to this article: http://dx.doi.org/10.3109/00016481809122018

曲 Published online: 08 Jul 2009.

Submit your article to this journal $\pi$

Џlll Article views: 1

Q View related articles $\sqsubset$ 


\title{
Uber Störungen im Harnsäure-Stoffwechsel bei Patienten mit Asthma bronchiale und Rhinitis vasomotoria.
}

\author{
Von A. de KLEYN und W. STORM van LEEUWEN.
}

Vor einigen Jahren hat Posthumus Meyes ${ }^{1}$ darauf hingewiesen, dass bei einer Anzahl Patienten, welche an Rhinitis vasomotoria leiden, Abweichungen im Harn gefunden werden. Untersuchte er nämlich bei diesen Patienten den Säuregehalt des Morgenharns, indem er denselben gegen $\mathrm{Na} \mathrm{OH}$ mit Phenolphthaleine als Indikator titrierte, so fand rr, dass derselbe viel höher war, als bei normalen Personen. Posthumus Meyes schrieb diesen Patienten eine bestimmte Diät vor und in vielen Fällen ging nach Anwendung dieser Diät die Rhinitis vasomotoria zurück und auch der Säuregehalt im Harn verminderte. Bei einer Anzahl Patienten von de Kleyn hatte eine gleiche Behandlung dasselbe Resultat. Da also die Annahme, dass bei Rhinitis vasomotoria Stoffwechselstörungen im Spiele seien, vor der Hand lag, wurde bei einer weiteren Reihe von Patienten, welche an typischen Erscheinungen von Rhinitis vasomotoria litten, eine ausgebreitetere Untersuchung verrichtet deren Zweck war, über die Art dieser Stoffwechsel-Störungen nähere Aufklärungen zu geben. Es schien erwünscht, nuch Asthmapatienten in dieser Richtung zu untersuchen. Hiervon macht Posthumus Meyes in seiner oben erwähnten Arbeit schon Erwähnung, während in der französischen Literatur wiederholt auf den Zusammenhang zwischen s. 581 .

Zeitschrift für Laryngologie, Rhinologie und ihre Grenzgebiete 1910, 
Asthma und Harnsäure-Stoffwechsel hingewiesen wird, ohne dass jedoch unseres Wissens dieser Zusammenhang bewiesen worden wäre:

Der erste Asthmapatient ist schon in 1911 untersucht worden; die Ausscheidung von Harnsäure hatte bei ihm einen sehr eigenartigen Verlauf. Normal betrug dieselbe ungefähr 300-400 Mgr. Kurz von einem Asthmaanfall verminderte sie um ein sehr beträchtliches, so dass sogar Werte von weniger als $100 \mathrm{Mgr}$. gefunden wurden. Während oder nach dem Anfall stieg die Ausscheidung über das Normale. Dreimal hintereinander konnten wir diese Erscheinung bei dem Patienten beobachten. Es war uns leider nicht möglich den. Patienten, der sich in häuslicher Pflege befand, zu veranlassen, sich streng an die purinfreie Diät zu halten, so dass aus dieser Untersuchung keine bestimmten Schlüsse gezogen werden können. Die Veränderungen in der Harnsäureausscheidung waren jedoch solcherart, dass sie uns veranlassten, bei den späteren Patienten dem Harnsäure-Stoffwechsel besondere Aufmerksamkeit zu schenken, umsomehr als Posthumus Meyes in seiner oben genannten Arbeit auf den Zusammenhang zwischen Gicht und Nasenleiden hinweist.

Im Ganzen sind 12 Patienten auf die folgende Weise untersucht worden: Die Patienten wurden einige Tage hindurch auf eine Diät gesetzt, welche so wenig möglich Purinstoffe enthielt. Einfachheitshalber soll diese Diät purinfreie Diät genannt werden. Dann wurde ihnen einen Tag lang sehr purinreiche Nahrung verabreicht und danach wieder einige Tage hindurch purinfreie Diät. Während des ganzen Verlaufs der Untersuchung wurde Sorge getragen dass die Menge von Nahrung, welche der Patient täglich zu sich nahm, möglichst die gleiche sei. Es wurde den Patienten überlassen wieviel Nahrung sie zu sich nehmen wollten, die Menge jedoch, welche sie am ersten Tage genommen hatten, wurde so genau wie möglich auch an

1 E. Delthil L'Asthme. Étiologie pathogénie, traitement. Son origine arthritique. Son antagonisme avec la tuberculose. Paris 1917. Mit ausgebreitetem frazzösischem Literaturverzeichnis. 
den folgenden Tagen gegeben. Diese Vorsorgsmassregel welche nicht allein für die feste, sondern auch für die flüssige Nahrung und Getränke beobachtet wurde, war deshalb erforderlich, weil grosse Unterschiede in der Menge, auch wenn dieselbe purinfrei ist, doch einen Einfluss ausüben können auf die Menge der Harnsäure, welche ausgeschieden wird (eine Steigung der endogenen Harnsäureausschneidung). Ausserdem wurden die Patienten gebeten, während der Dauer der Untersuchung so regelmässig wie möglich zu leben, jeden Tag zur gleichen Stunde aufzustehen, auszugrehen, u. s. w. Da es sich herausstellte, dass das Einhalten der Diät, das Aufbewahren des Harns u. s. f. nicht mit der erforderlichen Genauigkeit geschah, wurden alle Patienten ${ }^{1}$ während der Untersuchung in's Diakonissenhaus iufgenommen *.

Von jedem Patienten wurde der Harn von 24 Stunden in eine 2 Literflasche zusammengesammelt, wobei Sorge getragen wurde, dass der Harn unmittelbar nach der Miction in diese Flasche gegossen werde, um zu verhüten, dass sich in dem Gefäss, in welchem der Harn aufgefangen worden war, durch Abkühlung ein Niederschlag von Harnsäure bilde. An dem Tage, an welchem eine purinreiche Diät vorgeschrieben war, bekamen die Patienten ausser der gewöhnlichen Nahrung noch eine tüchtige Portion Fleisch, Niere oder Kalbsbries. In der letzten Zeit war immer $100 \mathrm{Gr}$. Fleisch und $100 \mathrm{Gr}$. Kalbsbries gegeben worden. Es stellte sich heraus, dass bei normalen Menschen hiernach ausser der gewöhnlichen Menge innerhalb zwei Tagen 6 bis $700 \mathrm{Mgr}$. Harnsäure extra ausgeschieden wird. Bei dem Harn von $2 \dot{4}$ Stunden wurde nun täglich bestimmt:

a) die Quantität und das specifische Gewicht

b) die Menge Stickstoff nach der Methode von Kjeldahl (Microbestimmung nach Bang)

${ }^{1}$ Mit Ausnahme von Pat. E. Mediziner.

2 Herrn Direktor Dr. Cramer mőge an dieser Stelle unser aufrichtiger Dank ausgesprochen werden far seine Mithilfe, mit welcher er die genaue Ausführung dieser Untersuchungen forderte. 


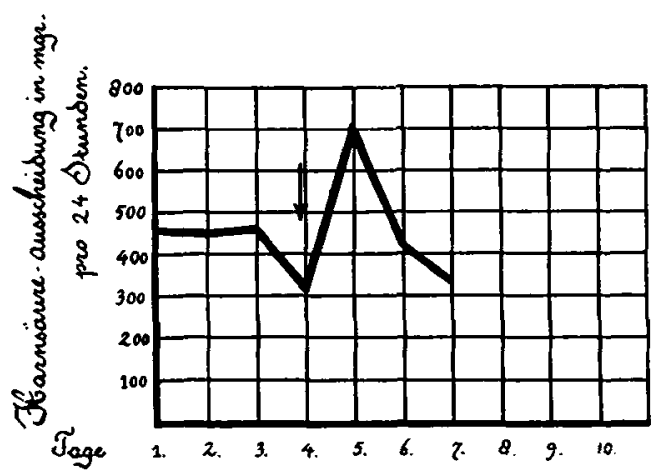

Fig. 1. Harnsäure-Ausscheidung von Pat. A (normal).

Bei $\downarrow 100$ gr. Fleisch + Kalbstries (gew. unbekannt).

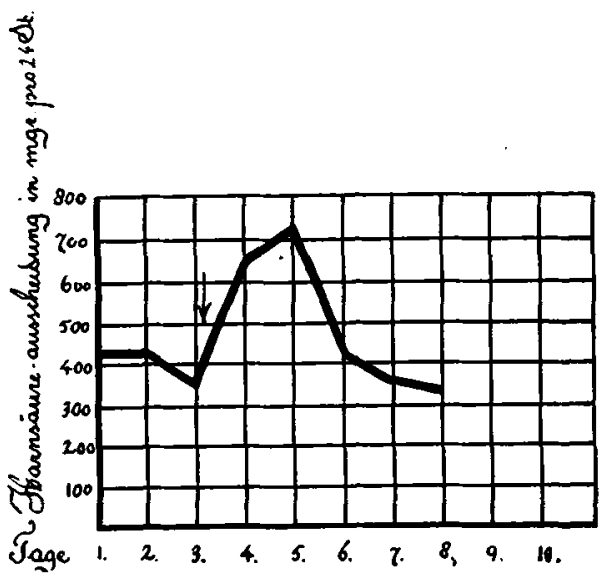

Fig. 2. Harnsăure-Ausscheidung von Pat. $D$ (normal).

Bei $\downarrow 100$ gr. Fleisch +100 gr. Kalbsbries extra. c) die Menge Kreatinine nach der colorimetrischen Methode von Autenrieth-Koenigsberger,

d) die Menge Harnsäure wach der Methode von Folin und Schaffer,

e) bei einem Teil der Patienten die MengeKochsalz nach der Methode von Volhardt.

In Tabelle I sind die Resultate sämtlicher Bestimmungen zusamm engestellt, während in Tabelle II angegeben ist, auf welche Weise bei den verschiedenen Patienten die Ausscheidung vom Übermass an Harnsäure (nach dem Tage, an welchem purinreiche Diät verabreicht worden war) erfolgt ist.

Bezüglich der Ausscheidung von Harnsäure sind die folgenden Beobachtungen gemacht worden. Bei 3 Patienten (A. D. und $\mathrm{H}$ ) verlief die Ausscheidung normal. Die erste Patientin (A) gehört nicht zu dieser Gruppe von Patienten, wurde jedoch in diese Tabelle aufgenommen, um den Verlauf bei einem normalen Menschen zu veranschaulichen. In Fig. 1 ist solch eine Purin- 
ausscheidung kurvenmässig dargestelt. Der zweite Patient, welcher an Asthma litt, hatte eine vollkommen normale Harnsäureausscheidung, wie auch aus der Kurve (Fig. 2) hervorgeht. Dass bei ihm keine Stoffwechselstörungen gefunden wurden, veranlasste uns, ihn nochmals genauer zu untersuchen, wobei sich herausstellte, dass die Anfälle bei ihm nicht so wie bei den meisten Asthmapatienten, mit Pfeifen in den feinen Verzweigungen der Luftwege gepaart waren, sondern dass das Pfeifen, welches man beim Auskultieren der Lungen hörte, seinen Ursprung im Kehlkopf hatte. Einem von uns (de Kleyn) ist es gelungen, während eines Anfalles zu laryngoscopisieren, wobei er beim Ausatmen ein krampfhaftes Zusammenziehen der Stimmbänder

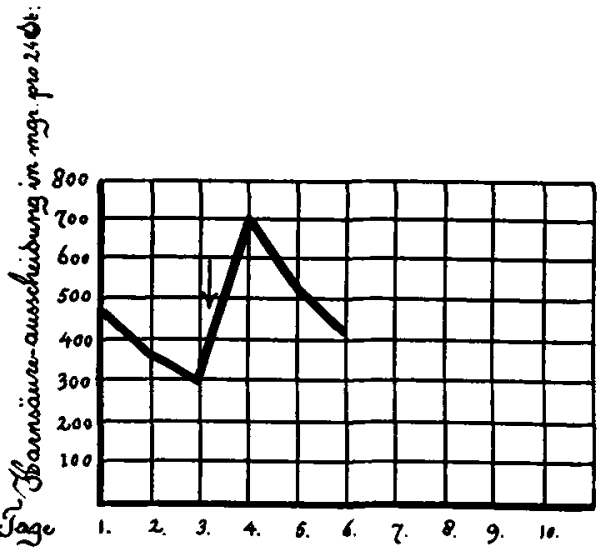

Fig. 3. Harnsaure-Ausscheidung von Pat. H. (Heufieber).

Bei $\downarrow 100$ gr. Fleisch +100 gr Kalbsbries extra.

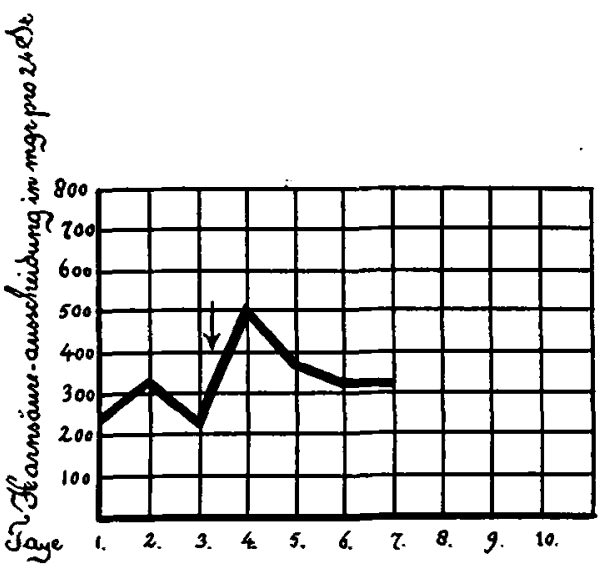

Fig. 4. Harnsãure-Ausscheidung von Pat. B. (Rhinitis).

Bei $\downarrow 100$ gr. Fleisch +100 gr. Kalbsbries extra.

beobachtete. Die Stimmbänder waren mit eingetrocknetem Schleim bedeckt, sobald dieser entfernt wurde, hörte der Anfall auf. Dies ist also ein Fall von Asthma wie schon Talma ${ }^{1}$

1 (Berl. klin. Wochenschrift 1898 n:r 52). 


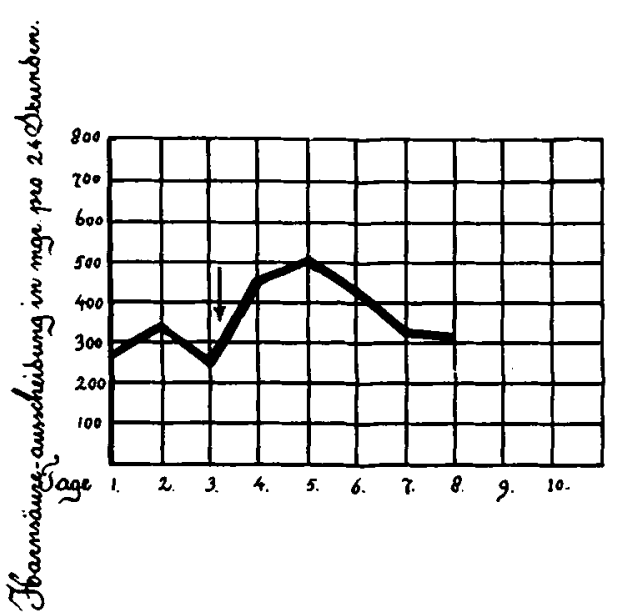

Fig. 5. Harnsäure-Ausscheidung von Pat. K. (Asthma).

Bei $\downarrow 100$ gr. Fleisch +100 gr. Kalbsbries extra.

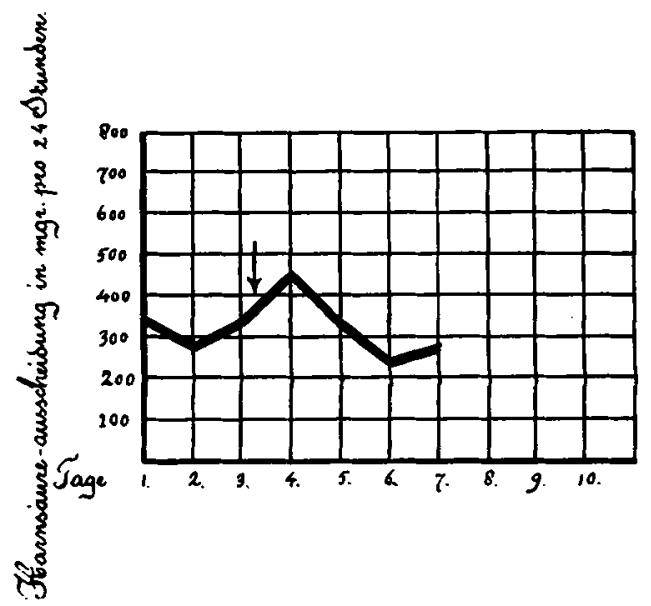

Fig. 6. Harnsaure-Ausscheidung von Pat. C. (Asthma).

Bei $\downarrow 100$ gr. Fleisch +100 gr. Kalbsbries extra. gegeben wird von der Harnsäureausscheidung von B (Rhinitis) und $K$, Asthmapatienten.

im J. 1898 beschrieben hat. Der dritte, Patient (H.) hatte Heufieber (Purinausscheidung s. Fig. 3).

Bei einer nicht in die Tabelle aufgenommenen, übrigens zweifelhaften Rhinitis-vasomotoriapatientin wurden keine Störungen in der Ausscheidung der Harnsäure gefunden. Die übrigen Patienten können in zwei Gruppen verteiltwer. den. Bei der ersten Gruppe (hierzu müssen die Patienten $\mathbf{B}$, $K$ und $E$ in der ersten Periode gezählt werden) wurde nach der purinreichen Nahrung der Überschuss an Harnsäure wohl ausgeschieden, es dauerte jedoch 3 bis 4 Tage, also länger als bei normalen Menschen. Dies ist deutlich zu ersehen aus Fig. 4 und 5, in denen eine schematische Vorstellung$$
\text { Dis) und } \mathrm{K} \text {, Asthmapatienten. }
$$ 


\begin{tabular}{|c|c|c|c|c|c|c|c|c|}
\hline 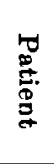 & Datum & 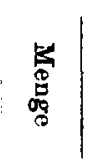 & $\begin{array}{l}\square \\
9 \\
0 \\
? \\
4\end{array}$ & $\begin{array}{l}2 \\
5 \\
g \\
g\end{array}$ & 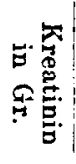 & 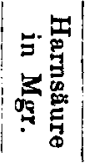 & 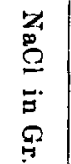 & Bemerkungen. \\
\hline A. & $\mid \begin{array}{r}29-2-1916 \\
30-2-1916 \\
2-3-1916 \\
3-3-1916 \\
4-3-1916 \\
5-3-1916 \\
6-3-1916\end{array}$ & $\begin{array}{ll}1 & 310 \\
1 & 050 \\
1 & 150 \\
1 & 110 \\
2 & 300 \\
1 & 260 \\
1 & 050\end{array}$ & $\begin{array}{l}\bar{z} \\
\bar{z} \\
\overline{-} \\
-\end{array}$ & $\begin{array}{l}9.4 \\
= \\
= \\
= \\
-\end{array}$ & $\begin{array}{l}1.27 \\
1.07 \\
1.15 \\
0.8 \\
1.5 \\
0.95 \\
0.97\end{array}$ & $\begin{array}{l}487 \\
464 \\
485 \\
322 \\
731 \\
423 \\
352\end{array}$ & $\begin{array}{l}\overline{-} \\
\overline{-} \\
\overline{-} \\
\overline{-}\end{array}$ & $\left\{\begin{array}{c}100 \text { gr. Fleiseh }+ \\
\text { Kalbsbries (Gew. } \\
\text { unbekannt). }\end{array}\right.$ \\
\hline D. & $\begin{array}{r}5-2-1917 \\
6-2-1917 \\
7-2-1917 \\
8--2-1917 \\
9-2-1917 \\
10-2-1917 \\
11-2-1917 \\
12-2-1917\end{array}$ & $\left|\begin{array}{rr}1 & 120 \\
9100 \\
1 & 210 \\
1 & 240 \\
1 & 200 \\
840 \\
1 & 110 \\
930\end{array}\right|$ & $\begin{array}{ll}1 & 020 \\
1 & 025 \\
1 & 023 \\
1 & 021 \\
1 & 020 \\
1 & 023 \\
1 & 020 \\
1 & 024\end{array}$ & $\begin{array}{r}9.7 \\
9.3 \\
10.7 \\
11.6 \\
11.9 \\
10.8 \\
10.1 \\
11,5\end{array}$ & $\begin{array}{l}1.0 \\
1.2 \\
1.2 \\
1.2 \\
1.2 \\
1.2 \\
1.1 \\
1.1\end{array}$ & $\begin{array}{l}432.6 \\
432 \\
363 \\
651 \\
715 \\
412 \\
370 \\
326\end{array}$ & $\begin{array}{r}7.8 \\
7.6 \\
13.0 \\
12.9 \\
16.0 \\
8.2 \\
11.8 \\
10.5\end{array}$ & $\left\{\begin{array}{l}100 \text { gr. Kalbsbrie } \\
+100 \text { gr. Fleisch }\end{array}\right.$ \\
\hline $\mathbf{H}$. & $\begin{array}{l}15-5-1917 \\
16-5-1917 \\
17-5-1917 \\
18-5-1917 \\
19-5-1917 \\
20-5-1917 \\
21-5-1917 \\
22-5-1917\end{array}$ & $\begin{array}{ll}2 & 300 \\
2 & 400 \\
2 & 480 \\
2 & 020 \\
2 & 610 \\
1 & 650 \\
1 & 205 \\
1 & 130\end{array}$ & 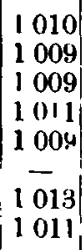 & $\begin{array}{l}11.6 \\
11.0 \\
11.3 \\
13.3 \\
12.2 \\
12.3 \\
9.3 \\
8.0\end{array}$ & $\begin{array}{l}1.3 \\
1.5 \\
1.3 \\
1.3 \\
1.2 \\
1.2 \\
1.2 \\
1.1\end{array}$ & $\begin{array}{l}483 \\
360 \\
297 \\
759 \\
512 \\
407 \\
324 \\
194\end{array}$ & $\begin{array}{l}- \\
\overline{-} \\
\overline{-} \\
8.8 \\
7.3 \\
6.2 \\
4.3\end{array}$ & $\mid \begin{array}{l}17-7-1917 \\
100 \text { gr. Fleisch }+ \\
100 \text { gr. Kalbsbries. }\end{array}$ \\
\hline C & $\begin{array}{l}17-1-1917 \\
18-1-1917 \\
19-1-1917 \\
20-1-1917 \\
21-1-1917 \\
22-1-1917 \\
23-1-1917 \\
24-1-1917 \\
25-1-1917 \\
26-1-1917 \\
27-1-1917 \\
28-1-1917 \\
29-1-1917\end{array}$ & $\begin{array}{l}680 \\
780 \\
700 \\
440 \\
550 \\
830 \\
710 \\
700 \\
900 \\
730 \\
520 \\
530 \\
690\end{array}$ & $\begin{array}{ll}1 & 021 \\
1 & 021 \\
1 & 024 \\
1 & 030 \\
1 & 030 \\
1 & 028 \\
1 & 029 \\
1 & 025 \\
1 & 021 \\
1 & 028 \\
1 & 032 \\
1 & 031 \\
1 & 027\end{array}$ & $\begin{array}{r}7.3 \\
8.7 \\
8.5 \\
7.6 \\
8.3 \\
1.5 \\
10.7 \\
8.9 \\
9.3 \\
9.1 \\
9.0 \\
9.2 \\
10.1\end{array}$ & $\begin{array}{l}0.54 \\
0.53 \\
0.47 \\
0.42 \\
0.41 \\
0.52 \\
0.44 \\
0.43 \\
0.47 \\
0.44 \\
0.44 \\
0.4 \\
0.47\end{array}$ & $\begin{array}{c}295 \\
271 \\
341 \\
304 \\
- \\
- \\
330 \\
288.7 \\
330 \\
449 \\
317 \\
226 \\
280\end{array}$ & $\begin{array}{l}7.0 \\
6.7 \\
5.4 \\
4.0 \\
5.5 \\
8.1 \\
6.4 \\
6.4 \\
7.6 \\
8.5 \\
4.9 \\
5.0 \\
6.6\end{array}$ & $\left\{\begin{array}{l}100 \text { gr. Kalbsbri } \\
+100 \text { gr. Fleiscl }\end{array}\right.$ \\
\hline E. & $\begin{array}{r}20-2-1917 \\
21-2-1917 \\
22-2-1917 \\
23-2-1917 \\
24-2-1917 \\
25-2-1917 \\
26-2-1917 \\
27-2-1917 \\
28-2-1917 \\
1-3-1917\end{array}$ & \begin{tabular}{|r}
1030 \\
1560 \\
1200 \\
950 \\
680 \\
870 \\
700 \\
1330 \\
1370 \\
580
\end{tabular} & $\begin{array}{ll}1 & 020 \\
1 & 013 \\
1 & 013 \\
1 & 028 \\
1 & 033 \\
1 & 030 \\
1 & 027 \\
1 & 026 \\
1 & 014 \\
1 & 029\end{array}$ & $\begin{array}{r}10.2 \\
7.9 \\
7.7 \\
9.8 \\
11.2 \\
12.3 \\
10.3 \\
14.1 \\
13.8 \\
8.5\end{array}$ & $\begin{array}{l}1.4 \\
1.8 \\
1.2 \\
1.2 \\
1.3 \\
1.3 \\
1.2 \\
1.8 \\
1.3 \\
1.1\end{array}$ & $\begin{array}{l}459 \\
351 \\
405 \\
678 \\
606 \\
515 \\
375 \\
638 \\
462 \\
404\end{array}$ & \begin{tabular}{|r|}
10.3 \\
8.7 \\
6.6 \\
11.8 \\
9.5 \\
10.3 \\
8.0 \\
15.6 \\
9.1 \\
7.1
\end{tabular} & $\left\{\begin{array}{l}100 \text { gr. Fleisch }+ \\
100 \text { gr. Kalbsbries. }\end{array}\right.$ \\
\hline
\end{tabular}




\begin{tabular}{|c|c|c|c|c|c|c|c|c|}
\hline 㿣 & Datum & 椱 & प़ & $\begin{array}{l}2 \\
5 \\
5 \\
9\end{array}$ & 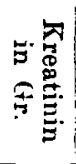 & 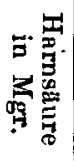 & 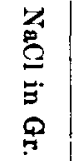 & Bemerkungen. \\
\hline E. & $\begin{array}{l}2-3-1917 \\
3-3-1417 \\
4-3-1917 \\
5-3-1917 \\
6-3-1917 \\
7-3-1917 \\
8-3-1917\end{array}$ & $\left|\begin{array}{rr}1 & 180 \\
1 & 000 \\
1 & 430 \\
980 \\
630 \\
810 \\
1 & 100\end{array}\right|$ & $\begin{array}{ll}1 & 018 \\
1 & 027 \\
1 & 018 \\
1 & 019 \\
- & -\end{array}$ & $\begin{array}{r}9.2 \\
11.3 \\
12.1 \\
9.5 \\
- \\
-\end{array}$ & $\begin{array}{l}1.2 \\
1.3 \\
1.1 \\
1.1 \\
1.2 \\
1.1 \\
1.3\end{array}$ & $\begin{array}{l}451 \\
629 \\
386 \\
404 \\
413 \\
410 \\
486\end{array}$ & \begin{tabular}{r|}
10.9 \\
15.4 \\
11.7 \\
7.9 \\
$=$ \\
-
\end{tabular} & $\left\{\begin{array}{l}\text { Am Abend gerin- } \\
\text { ges Asthma. } \\
100 \text { gr. Fleisch } \\
100 \text { gr. Kalbsbries. } \\
\text { Leicht asth. } \\
\text { matisch. }\end{array}\right.$ \\
\hline F. & $\begin{array}{r}8-3-1917 \\
9-3-1917 \\
10-3-1017 \\
11--3-1917 \\
12-3-1917 \\
13-3-1917 \\
14-3-1917 \\
15-3-1917\end{array}$ & $\mid \begin{array}{rr}1 & 620 \\
730 \\
1000 \\
730 \\
1150 \\
1110 \\
730 \\
1000\end{array}$ & $\begin{array}{ll}1 & 027 \\
1 & 026 \\
1 & 025 \\
1 & 026 \\
1 & 017 \\
1 & 015 \\
1 & 022\end{array}$ & $\begin{array}{r}13.7 \\
6.9 \\
9.6 \\
7.7 \\
11.0 \\
7.7 \\
6.8 \\
8.1\end{array}$ & $\begin{array}{l}1.5 \\
0.66 \\
0.96 \\
0.73 \\
1.1 \\
0.8 \\
0.96 \\
0.99\end{array}$ & $\begin{array}{l}522 \\
287 \\
367 \\
394 \\
496 \\
283 \\
334 \\
300\end{array}$ & $\begin{array}{r}24.6 \\
11.1 \\
12.9 \\
9.3 \\
11.3 \\
9.9 \\
7.7 \\
10.3\end{array}$ & $\left\{\begin{array}{l}11-3-19 \\
100 \mathrm{gr} . \mathrm{Fl} \\
100 \mathrm{gr} . \mathrm{Ka}\end{array}\right.$ \\
\hline $\mathbf{K}$. & $\begin{array}{r}9-7-1917 \\
10-7-1917 \\
11-7-1917 \\
12-7-1917 \\
13-7-1917 \\
14-7-1917 \\
15-7-1917 \\
16-7-1917 \\
17-7-1917\end{array}$ & $\begin{array}{r}1150 \\
970 \\
1130 \\
1270 \\
750 \\
680 \\
1640 \\
930 \\
1480\end{array}$ & $\begin{array}{ll}1 & 018 \\
1 & 020 \\
1 & 020 \\
1 & 015 \\
1 & 026 \\
1 & 029 \\
1 & 015 \\
1 & 020 \\
1 & 022\end{array}$ & $\begin{array}{r}8.3 \\
7.7 \\
9.1 \\
9.6 \\
8.0 \\
10.1 \\
13.0 \\
8.6 \\
10.3\end{array}$ & $\begin{array}{l}1.1 \\
1.1 \\
1.1 \\
1.1 \\
1.1 \\
1.1 \\
1.4 \\
1.2 \\
1.2\end{array}$ & $\begin{array}{l}310 \\
276 \\
318 \\
248 \\
466 \\
505 \\
424 \\
327 \\
310\end{array}$ & \begin{tabular}{|r|}
11.0 \\
8.9 \\
9.1 \\
8.6 \\
8.3 \\
8.3 \\
12.1 \\
8.6 \\
22.5
\end{tabular} & $\left\{\begin{array}{l}12-7-1917 . \\
100 \text { gr. Fleisch }+ \\
100 \text { gr. Kalbsbries. }\end{array}\right.$ \\
\hline B. & $\begin{array}{l}15-6-1916 \\
16-6-1916 \\
17-6-1916 \\
18-6-1916 \\
19-6-1916 \\
20-6-1916 \\
91-6-1916\end{array}$ & $\begin{array}{r}980 \\
630 \\
730 \\
1170 \\
1430 \\
1800 \\
1030\end{array}$ & $\begin{array}{c}1 \overline{017} \\
= \\
= \\
=\end{array}$ & $\begin{array}{r}7.7 \\
6.1 \\
7.8 \\
1.2 \\
9.7 \\
11.0\end{array}$ & $\begin{array}{l}0.87 \\
0.41 \\
0.82 \\
1.0 \\
0.94 \\
1.1 \\
0.92\end{array}$ & $\begin{array}{l}232 \\
316 \\
219 \\
496 \\
386 \\
324 \\
324\end{array}$ & $\begin{array}{l}\bar{z} \\
\bar{z} \\
\bar{z}\end{array}$ & $\left\{\begin{array}{l}100 \text { gr. Fleisch }+ \\
100 \text { gr. Kalbsbries. }\end{array}\right.$ \\
\hline G. & $\begin{array}{r}3-5-1917 \\
4-5-1917 \\
5-5-1917 \\
6-5-1917 \\
7-5-1917 \\
8-5-1917 \\
9-5-1917 \\
10-5-1917\end{array}$ & $\begin{array}{r}820 \\
710 \\
750 \\
900 \\
810 \\
900 \\
1090 \\
1011\end{array}$ & $\begin{array}{ll}1 & 016 \\
1 & 018 \\
1 & 016 \\
1 & 016 \\
1 & 020 \\
1 & 018 \\
1 & 013 \\
1 & 011\end{array}$ & $\begin{array}{l}6.8 \\
5.9 \\
5.1 \\
5.5 \\
6.7 \\
7.8 \\
6.4 \\
6.0\end{array}$ & $\begin{array}{l}084 \\
0.83 \\
0.67 \\
0.75 \\
0.73 \\
0.79 \\
0.75 \\
0.76\end{array}$ & $\begin{array}{l}301 \\
449 \\
196 \\
229 \\
380 \\
468 \\
278 \\
238\end{array}$ & $\begin{array}{l}- \\
- \\
- \\
- \\
-\end{array}$ & $\left\{\begin{array}{l}6-5-1917 \\
100 \text { gr. Fleisch }+ \\
100 \text { gr. Kalbsbries. }\end{array}\right.$ \\
\hline L. & $\begin{array}{l}24-5-1916 \\
25-5-1916 \\
26-5-1916 \\
27-5-1916 \\
28-5-1916\end{array}$ & $\begin{array}{r}1320 \\
860 \\
1200 \\
1120 \\
1090\end{array}$ & $\begin{array}{l}- \\
z \\
-\end{array}$ & $\begin{array}{r}6.7 \\
9.5 \\
11.0 \\
11.0 \\
16.0\end{array}$ & $\begin{array}{l}1.2 \\
1.8 \\
1.2 \\
1.1 \\
1.4\end{array}$ & \begin{tabular}{|l}
460 \\
370 \\
360 \\
468 \\
163
\end{tabular} & $\begin{array}{l}z \\
z \\
z\end{array}$ & $\left\{\begin{array}{l}\$ 50 \text { gr. Fleisch }+ \\
150 \text { gr. Niere. }\end{array}\right.$ \\
\hline
\end{tabular}




\begin{tabular}{|c|c|c|c|c|c|c|c|c|}
\hline 总 & Datum & 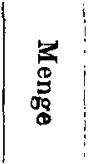 & 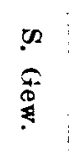 & $\begin{array}{l}7 \\
\square \\
8 \\
9\end{array}$ & 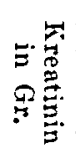 & 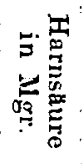 & 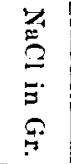 & Bemerkungen. \\
\hline L. & $\begin{array}{l}29-5-1916 \\
30-5-1916 \\
31-5-1916\end{array}$ & $\left|\begin{array}{ll}1 & 420 \\
1 & 320 \\
1 & 320\end{array}\right|$ & $\bar{z}$ & $\begin{array}{l}16.0 \\
14.0 \\
17.0\end{array}$ & $\begin{array}{l}1.1 \\
1.2 \\
1.2\end{array}$ & $\begin{array}{l}468 \\
455 \\
445\end{array}$ & $\begin{array}{l}- \\
-\end{array}$ & \\
\hline M. & $\begin{array}{l}20-5-1916 \\
21-5-1916 \\
22-5-1916 \\
23-5-1915 \\
24-5-1916 \\
25-5-1916 \\
26-5-1916 \\
27-5-1916\end{array}$ & $\left|\begin{array}{rr|}1 & 100 \\
1 & 080 \\
900 \\
850 \\
1 & 100 \\
1 & 150 \\
720 \\
1 & 110\end{array}\right|$ & $\begin{array}{l}\overline{-} \\
\overline{-} \\
\dot{-} \\
\overline{-} \\
-\end{array}$ & $\begin{array}{r}12.0 \\
12.7 \\
11.0 \\
15.9 \\
15.3 \\
9.4 \\
12.9\end{array}$ & \begin{tabular}{l|}
1.3 \\
1.27 \\
1.2 \\
1.3 \\
1.5 \\
1.33 \\
0.87 \\
1.25
\end{tabular} & $\begin{array}{l}519 \\
316 \\
337 \\
567 \\
561 \\
437 \\
410 \\
466\end{array}$ & $\begin{array}{l}- \\
z \\
- \\
- \\
-\end{array}$ & $\left\{\begin{array}{l}200 \mathrm{gr} \text {. Fleisch + } \\
130 \mathrm{gr} \text {. Niere extra. }\end{array}\right.$ \\
\hline I. & $\begin{array}{r}3-7-1917 \\
4-7-1917 \\
5-7-1917 \\
6-7-1917 \\
8-7-1917 \\
9-7-1917 \\
10-7-1917 \\
11-7-1917 \\
12-7-1917\end{array}$ & $\begin{aligned} 1570 \\
1120 \\
1510 \\
1500 \\
820 \\
1300 \\
1490 \\
1420 \\
780\end{aligned}$ & $\begin{array}{ll}1 & 016 \\
1 & 023 \\
1 & 016 \\
1 & 016 \\
1 & 026 \\
1 & 020 \\
1 & 016 \\
1 & 018 \\
1 & 028\end{array}$ & $\begin{array}{r}10.7 \\
9.8 \\
10.5 \\
10.0 \\
10.2 \\
12.8 \\
10.6 \\
11.1 \\
9.2\end{array}$ & $\begin{array}{l}1.2 \\
1.1 \\
1.2 \\
1.2 \\
1.0 \\
1.2 \\
1.2 \\
1.1 \\
1.2\end{array}$ & $\begin{array}{l}388 \\
453 \\
340 \\
438 \\
485 \\
585 \\
42.5 \\
420 \\
392\end{array}$ & $\begin{array}{r}12.7 \\
11.3 \\
11.1 \\
10.6 \\
5.2 \\
10.1 \\
11.3 \\
11.9 \\
8.3\end{array}$ & $\left\{\begin{array}{l}7-7-1917 \\
100 \text { gr. Fleisch }+ \\
100 \text { gr. Kalbsbries. }\end{array}\right.$ \\
\hline
\end{tabular}

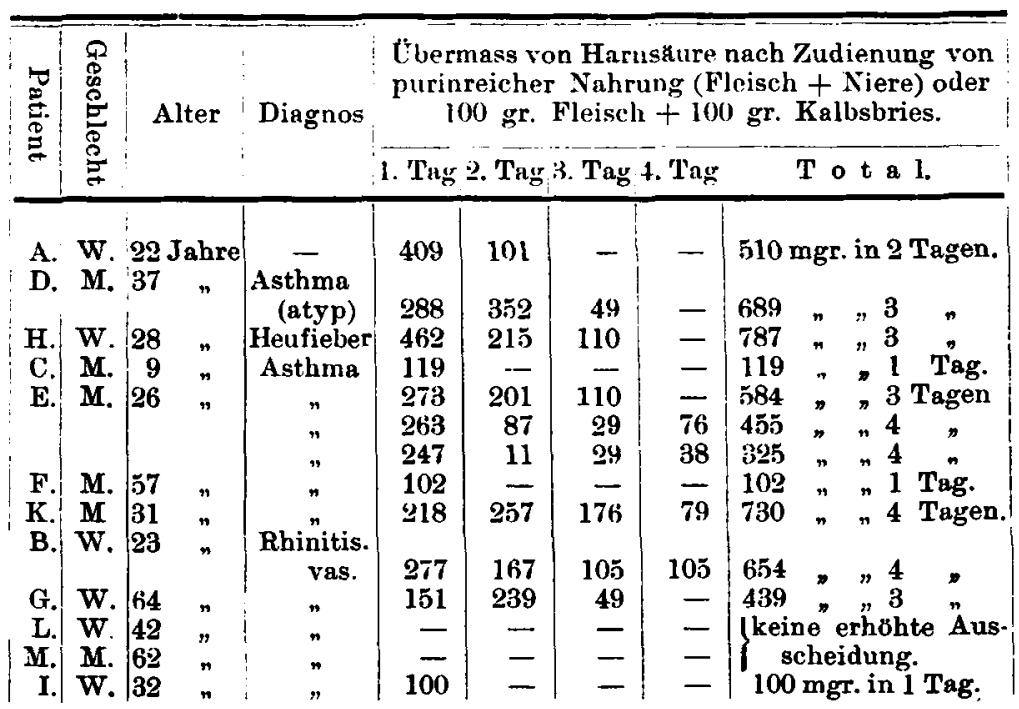


Bei der zweiten Gruppe (hierzu gehören C F L M I) wurde der Überschuss an Harnsäure während der ersten Tage gar nicht oder nur teilweise ausgeschieden (s. Fig. 6, 7 und 8).

In dieser Beziehung ist also zwischen der Ausscheidung der Harnsäure bei unseren Patienten und bei Gichtkranken

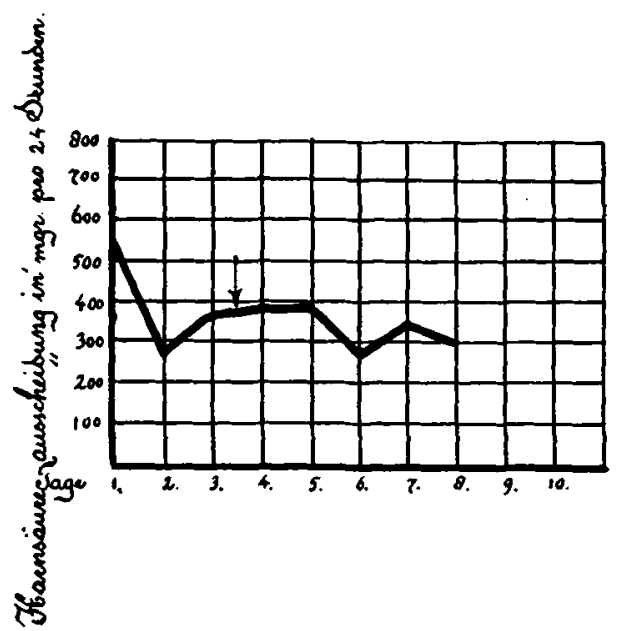

Fig. 7. Harnsäure-Ausscheidung ron Pat. F. (Asthma).

Bei $\downarrow 100$ gr. Fleisch +100 gr. Kalbsbries extra. eine Übereinstimmung zu sehen. Auch bei letzteren wird, wenn nach einerPeriode von purinfreierNahrung an einem Tage purinreiche Diät verabreicht wird, der Überschuss an Harnsäureentwedergarnicht oder erst im Laufe von 3 bis 4 Tagen, also langsamer als bei normalen Menschen, ausgeschieden. Was die Ursache dieser Erscheinung ist, ob weniger Harnsäure gebildetwird oder ob - was wahrscheinlicher ist - ein

Teil der Harnsäure im Körper festgehalten wird, ist weder für Gicht, noch für unsere Fälle mit Sicherheit festgestellt. Ein enger Zusammenhang zwischen Harnsäureausscheidung und der Heftigkeit oder der Frequenz der Asthmaanfälle konnte nicht nachgewiesen werden. Patient E. hat wohl einige Male gerade an Tagen, an welchen die Harnsäureausscheidung ętwas höher gewesen war als an anderen, behauptet, einen Asthmaanfall gehabt zu haben. Merkwürdig ist, dass Pat. E in einer Periode, während welcher er sich wohl fühlte, das Plus an Harnsäure ziemlich gut ausschied, während er typische Störungen hatte in einer späteren Periode (2:e Periode der Untersuchungsreihe) während welcher er auch asthmatisch war und nachts Asthmapulver brennen musste. D. Klinkert 
rechnet in einer sehr bemerkenswerten Mitteilung die Heufieberpatienten auch zu der "Gicht"-Gruppe. Patientin $\mathrm{H}$, die auch an Heufieber litt, hatte vollkommen normale Harnsäureausscheidung, allerdings wurde sie ausserhalb ihrer Heufieberperiode untersucht. Da man jedoch aus einem einzigen Fall keine Schlüsse ziehen kann, so muss es der Untersuchung einer grösseren Zahl von Fällen vorbehalten bleiben zu entscheiden ob das Heufieber in diese Gruppe gehöre oder nicht.

Die Kreatininausscheidung war bei sämmtlichen Patienten während der ganzen Dauer der Untersuchung nahezu gleichförmig. Die Stick-

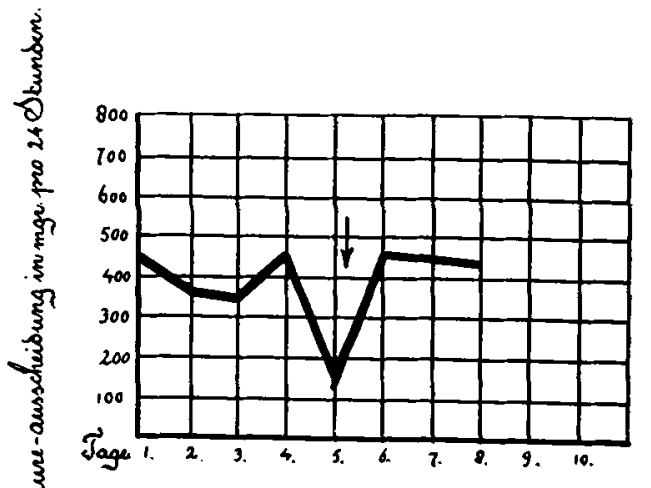
stoff- und Kochsalzausscheidung war jedoch sehr wechselnd, ohne dass hieraus irgendwelche bestimmte Schlüsse gezogen hätten werden können. Als Merkwürdigkeit seien nur die grossen Unterschiede in der Ausscheidung von $\mathrm{NaCl}$ bei $\mathrm{C}$ und I erwähnt.

Bei der Untersuchung stellte sich also heraus, dass mit Ausnahme von Pat. A, der in dieser Beziehung als normal betrachtet werden muss, von Pat. H., der an Heufieber litt und von Pat. D., der eine eigenartige Form von Asthma hatte, bei sämmtlichen Patienten eine Abweichung in der Ausscheidung der Harnsäure festgestellt werden konnte. Nun ist die Frage naheliegend, inwieferne dieser Befund für die Behandlung von Wichtigkeit ist. Es muss gleich bemerkt werden, dass die Resultate einer Behandlung, welche von diesen Befunden ausgeht, bei Asthma viel weniger günstig sind, als bei Rhinitis vasomotoria. Keines- 
falls kann behauptet werden, dass ein Asthmakranker durch das Einhalten einer purinfreien Diät von seinen Anfällen befreit werder kann. Pat. E. und F. hatten während der purinfreien Periode weniger und weniger heftige Anfälle als vorher; inwieferne dies mit der Diät im Zusammenhang steht, ist schwierig zu bestimmen. Pat. F., der an sehr heftigen Asthmaanfällen litt, weigerte sich, die purinfreie Diät länger als einige Wochen einzuhalten und Pat. E. bekam, nachdem es ihm bei purinfreier Diät monatelang ausgezeichnet gegangen war, schliesslich doch noch einen Anfall ${ }^{1}$, der ziemlich heftig war, ausserdem hatte er auch früher schon Perioden mit nur wenigen, schwachen Anfällen gehabt. Bei Pat. C. wurde bei einer Sputumuntersuchung, die während der Stoffwechseluntersuchung ausgeführt worden war, eine geringe Zahl Tuberkelbacillen gefunden; nach Diät und antitub. Behandlung ist das Asthma gänzlich verschwunden. Das Asthma von Pat. K. war nur leichtgradig, er hat jedoch erst kurze Zeit die purinfreie Diät eingehalten, so dass man sich über den Erfolg noch kein Urteil bilden kann.

Angesichts der grossen Übereinstimmung in der HarnsäureAusscheidung bei Gichtkranken und den hier beschriebenen Patienten, lag es vor der Hand, einen Versuch mit Atophan zu machen. Pat. F. reagierte darauf, was die Ausscheidung von Harnsäure betrifft, in der gewohnten Weise, $d . h$. während der ersten Tage wurde mehr Harnsäure ausgeschieden, während nach Weglassen des Atophans eine starke Verminderung eintrat. Er gab an, während der ersten, dreitägigen Atophanperiode weniger unter Asthma gelitten zu haben, es ist jedoch schwierig, zu entscheiden, welche Rolle hierbei die Suggestion gespielt hat. Pat. E. bekam unmittelbar nach Verabreichung von Atophan einen heftigen Anfall (ähnliches kommt auch bei Gicht vor) hatte darnach jedoch während 4 Monaten überhaupt keinen Anfall, was seit Jahren nicht mehr geschehen war. Wir

1 Spater ist es ihm bez. seiner Asthmaanfälle sehr gut gegangen. Er hat so viel wie moglich purinfreie Diat eingehalten und immer sobald $\in \mathrm{r}$ das Herannahen eines Anfalls spürte Calcium genommen. 
haben bei ihm von einer weiteren Atophanverabreichung abgesehen.

Viel günstigere Erfolge wurden bei der Beliandlung von Rhinitis vasomotoria erzielt, wie auch schon nach der Untersuchung von Posthumus Meyes vorausgesetzt werden konnte. Pat, L. und M., die beide an sehr heftiger Rhinitis vasomotoria litten, welche sie bei Ausübung ihres Berufes sehr hinderte, merkten beide nach Einhaltung einer purinfreien Diät eine deutliche Besserung, die jetzt schon ein Paar Jahren anhält, obwohl von einer vollständigen Genesung, selbst nach wiederholter Verabreichung von Calcium, keine Rede sein konnte, eine noch deutlichere Besserung trat bei diesen 2 Patienten nach Atophan auf. Suggestion kann in diesen beiden Fällen höchst wahrscheinlich ausgeschlossen werden, da vorher eine ganze Reihe verschiedener Arzneien keine Veränderung des Krankheitsbildes zu Stande bringen konnte. Infolge des hohen Preises von Atophan konnte die Verabreichung desselben nicht weiter fortgesetzt werden. Patientin H., die ebenfalls eine heftige Rhinitis hatte, war nach Diät und Atophan vollständig von ihrem Leiden befreit. Da das Befinden dieser Pat. stationär (3 Jahre lang) gut blieb und die Atophan-Behandlung bald eingestellt. während die purinfreie Diät fortgesetzt wurde, ist es anzunehmen, dass in diesem Fall die Diät grösseren Einfluss hatte, als das Atophan. Auch bei Pat. I waren die Resultate sehr günstig, bei Einhaltung der purinfreien Diät sind alle Beschwerden des Pat. seit einem Jahr geschwunden.

Bei anderen, nicht in dieser Mitteilung aufgenommenen Rhinitis vasomotoria-Patienten wurde mit der Einhaltung von purinfreier Diät, meistens kombiniert mit Verabreichung von Calcium-Chlorid, in der Regel eine bedeutende Besserung, manchmal ein gänzliches Verschwinden der Beschwerden erzielt, wie auch Posthumus Meyes änhliches schon beschrieben hat. Die von ihm vorgeschriebene Diät weicht in soferne von der unserigen ab, dass er kleine Mengen Fleisch und Fisch gestattet, während wir eine möglichst purinarme Diät gaben. Ebenso wie Posthumus Meyes Acta oto-laryngologica. 
haben auch wir saure Speisen und Getrïnke vermeiden lassen.

Im Verlaufe dieser Untersuchung wurden also bei 2 Gruppen von Patienten deutliche Abweichungen im Harnsäurestoffwechsel grefunden. Selbstverständlich ist durch die Feststellung dieser Abweichung die Pathogenese von Asthma und Rhinitis vasomotoria nur zum Teil aufgeklärt. Diese Mitteilung trägt deshalb auch blos einen vorläufigen Charakter und ist geeignet die Grundlage einer weiteren experimentellen Untersuchung zu bilden.

Zum Schluss noch die Bemerkung, dass es sich bei der Aufnahme der Anamnese herausstellte, dass bei einigen Patienten Stoffwechselerkrankungen in der Familie vorkamen. So scheint in der Familie von Pat. C. sowohl väterlicherals mütterlicherseits Gicht aufgetreten zu sein. Merkwürdig ist, dass Patienten $\mathrm{H}$. die an Heufieber leidet und keine Störungen in der Purinausscheidung aufweist, angibt, dass ihr Vater an Gicht und eine Tante an Asthma leiden. Auch in den Familien von verschiedenen anderen AsthmaPatienten kam Gicht oder ein Leiden welches als Gicht bezeichnet wurde, mehrmals vor, derartige Fälle sind übrigens in der Litteratur schon beschrieben. 